\title{
Dissociative identity disorder: out of the shadows at last?
}

Antje A. T. S. Reinders and Dick J. Veltman

\section{Summary}

Dissociative identity disorder (DID) is a severely debilitating disorder. Despite recognition in the current and past versions of the DSM, DID remains a controversial psychiatric disorder, which hampers its diagnosis and treatment. Neurobiological evidence regarding the aetiology of DID supports clinical observations that it is a severe form of post-traumatic stress disorder.

\section{Keywords}

Aetiology; neurobiology; trauma; fantasy; PTSD; DID; childhood trauma; neuroimaging.

\section{Copyright and usage}

(c) The Author(s), 2020. Published by Cambridge University Press on behalf of the Royal College of Psychiatrists.
Antje A. T. S. (Simone) Reinders (pictured) is a senior researcher at the Department of Psychological Medicine, King's College London, UK. She leads research into the neurobiology of dissociative identity disorder. Dick J. Veltman is a psychiatrist and Professor of Neuroimaging at the Department of Psychiatry at Amsterdam UMC/NUmc, The Netherlands.

Dissociative identity disorder (DID) was first included in the DSM in 1980 (DSM-III) as 'multiple personality disorder' and it is a controversial psychiatric diagnosis. The controversy finds its roots in a debate regarding the aetiology of the disorder. Supporters of two diametrically opposed views have engaged in passionate debate for decades: ${ }^{1}$ the trauma model states that DID is a severe form of post-traumatic stress disorder (PTSD) originating in severe and chronic (childhood) traumatisation, whereas the fantasy model postulates that DID is predominately due to suggestion and enactment and is facilitated by high levels of fantasy proneness and suggestibility. Although the trauma versus fantasy debate has evolved and aetiological research has broadened in the past few decades, ${ }^{2}$ there are several reasons why the fantasy model continues to appeal to clinicians. One reason is that information in undergraduate and graduate textbooks about trauma and dissociation is inadequate or simply wrong, because (a) it is often based on experimental research in non-clinical samples, (b) it is not fully based on scientific research, (c) it contains unbalanced discussions about the detrimental impact of childhood traumatisation and (d) it disregards empirical evidence showing a relationship between dissociation and antecedent trauma. Another reason is reluctance to accept the nature and severity of childhood abuse that individuals with DID report. It is troubling and painful to acknowledge how common and devastating trauma is, especially chronic childhood abuse. Subconscious protective mechanisms can take over to deny the reality of such abuse (in a similar way as denying racism, the Holocaust, or global warming ${ }^{3}$ ) and to believe that DID is a factitious disorder, as stated by the fantasy model. ${ }^{1}$ However, it becomes increasingly apparent that severe childhood abuse, neglect and maltreatment are part of many psychiatric disorders and of our society. ${ }^{4}$

\section{(Mis)diagnosing DID}

The combination of insufficient training in recognising traumarelated dissociation, limited exposure to accurate scientific information about DID, symptom similarities with other disorders (such as schizophrenia, bipolar disorder and borderline personality disorder) and the aetiology debate has led to a reluctance to consider a diagnosis of DID, leading to under- and misdiagnosis of the disorder, hampering effective treatment. ${ }^{5}$ From the moment of seeking treatment for symptoms to the time of an accurate diagnosis of DID, individuals receive an average of four prior other diagnoses, inadequate pharmacological treatment, have several hospital admissions and consequently spend many years in mental health services. These years of misdirected treatment result in protracted personal suffering and high direct and indirect societal costs. Other factors contributing to under- and misdiagnosing of DID are unfamiliarity with the spectrum of dissociative disorders, the existence of feigned DID, the reluctance of individuals with DID to present their dissociative symptoms, often owing to feelings of shame, and lack of knowledge and appreciation of its epidemiology. DID has an estimated lifetime prevalence of around $1.5 \%,{ }^{5}$ meaning that at least one million people in the UK will suffer from DID during their life. It is therefore highly clinically relevant to move DID out of the abyss to facilitate earlier accurate diagnosis, prevent unnecessary suffering and promote research into faster and more targeted interventions.

\section{Neurobiological evidence for the trauma model of DID}

According to DSM-5 criteria, DID is characterised by, among other things, two or more distinct identities or personality states that coincide, with fluctuating consciousness and changing access to autobiographical memory. Personality-state-dependent brain activation was found for the first time in 1985 in a single patient at rest and has been confirmed in independent studies over time. In 2003, the first multi-participant stimulus-driven brain-imaging study revealed personality-state-dependent processing of neutral and trauma-related autobiographical memory scripts. In a followup study it was shown that individuals with DID can be distinguished from DID-simulating healthy controls with high and low levels of fantasy proneness. ${ }^{1}$ Importantly, these simulationindependent differences in brain activation patterns between different personality states in DID were replicated in an independent sample, altogether discrediting the fantasy model for DID. In DSM-5 a dissociative subtype for PTSD was included and the dissociative disorders were placed immediately after the trauma- and stress-related disorders, to suggest a close relationship between dissociative PTSD and DID. Research confirmed similarities in brain activation patterns during emotion overmodulation and undermodulation in an indirect comparison between the two. These neurobiological similarities between personality states in DID and PTSD subtypes support a trauma-related aetiology of DID. 
As regards neurostructural evidence, a smaller hippocampal volume is the most consistently reported neuroanatomical correlate of childhood traumatisation. Negative correlations between childhood maltreatment and hippocampal volume have been reported in both unmedicated individuals from the general community and transdiagnostically in people with psychiatric disorders. Chalavi and colleagues ${ }^{6}$ built on this evidence to study hippocampal global and subfield volumes in PTSD and DID in relation to childhood traumatisation with the aim of directly testing the trauma model for DID. They found a negative correlation between hippocampal volumes and childhood traumatisation across the two disorders, thereby providing neuroanatomical evidence for the clinical observations that DID is related to (severe) childhood trauma. This finding is particularly important because neuroanatomical data are unlikely to be subject to cognitive manipulation. Hence, these findings support the notion that DID is closely related to PTSD, as indicated by its placement in DSM-5, especially when childhood trauma is involved, and provide evidence for the trauma model of DID. The finding that DID is related to environmental factors was further supported by a multicentre study ${ }^{7}$ that evaluated the neurodevelopmental origins of abnormal cortical morphology in DID. This study examined overall cortical volume and its two constitutes, that is, cortical thickness and surface area, in individuals with DID. It found that individuals with DID differed from controls on all three measures and provided evidence that non-genetic, environmental factors affect multiple aspects of brain development in DID. Negative associations between abnormal brain morphology and early childhood traumatisation were found as well.

Taken together, brain activation studies have validated the DSM-5 identity criterion of DID by showing the existence of two or more distinct personality states, each with their own distinct pattern of brain activation in response to autobiographical trauma-related information. Studies of brain structure in DID have shown that DID is not likely to be a neurodevelopmental disorder but that environmental factors, such as early childhood traumatisation, have an impact on brain morphology in DID.

\section{Considerations}

The aetiology of DID has been debated for decades, questioning the validity of DID as a diagnostic entity in the DSM. Given that neurobiological and other evidence ${ }^{2}$ supports the trauma model for DID, it remains unclear why the aetiology of DID is still controversial, because for most other major psychiatric disorders, such as psychosis, the aetiology is also insufficiently known without such detrimental impact on diagnostic detection, treatment and patient's quality of life. We therefore propose that, given the available neurobiological evidence, it is time to move DID out of the shadows and to consider it as a mainstream psychiatric disorder.

National and international training information and training opportunities are available to support clinicians in becoming more familiar with dissociation, dissociative symptoms, DID and dissociative disorders in general. The Trauma and Dissociation Service at South London and Maudsley NHS Foundation Trust, the Clinic for Dissociative Studies in London and the Pottergate Centre for Dissociation \& Trauma in Norwich are some of the national expertise centres in the UK. The European Society for Trauma and Dissociation (ESTD) and the International Society for the Study of Trauma and Dissociation (ISSTD) provide resources, information, training and advice to develop and promote comprehensive, clinically effective and empirically based responses to trauma and dissociation.

A final consideration is to employ whole-brain structural brain imaging to aid the diagnosis of DID. Structural brain imaging holds the promise of using objective biomarkers at the individual level to facilitate a fast and correct diagnosis of individuals with DID. A first study using pattern recognition methodologies has shown that individuals with DID can be distinguished from healthy controls at an individual level with a degree of accuracy that is comparable to what has been demonstrated for most psychiatric disorders. ${ }^{5}$ Future studies are needed to enhance clinical relevance by replicating previous findings and by distinguishing between DID and other psychopathologies using these pattern recognition methods.

Moving DID out of the shadows of psychiatry will facilitate earlier accurate diagnosis, faster and more targeted interventions, prevent unnecessary direct and indirect societal costs, but most important of all prevent years of suffering for individuals with the disorder.

Antje A.T.S. Reinders (D), Centre for Affective Disorders, Department of Psychological Medicine, Institute of Psychiatry, Psychology and Neuroscience, King's College London, UK; Dick J. Veltman, Department of Psychiatry, Amsterdam University Medical Centers, Vrije Universiteit (VU) Medical Center, VU Amsterdam, The Netherlands

Correspondence: A.A.T.S. Reinders. Email: a.a.t.s.reinders@kcl.ac.uk

First received 12 Jun 2020, final revision 21 Aug 2020, accepted 5 Sep 2020

\section{Acknowledgement}

We thank Professor Bethany Brand and Professor Allan Young for suggestions on parts of the content.

\section{Author contributions}

A.A.T.S.R. conceptualized and wrote the first draft of the manuscript and D.J.V. provided feedback and review towards the final version. Both authors approve of the final version of the manuscript.

\section{Funding}

This editorial represents independent research part funded by the National Institute for Health Research (NIHR) Biomedical Research Centre at South London and Maudsley NHS Foundation Trust and King's College London. The views expressed are those of the authors and not necessarily those of the National Health Service (NHS), the NIHR or the Department of Health.

\section{Declaration of interest}

None.

CMJE forms are in the supplementary material, available online at https://doi.org/10.1192 bjp.2020.168.

\section{References}

1 Reinders AATS, Willemsen ATM, Vos HPJ, den Boer JA, Nijenhuis ERS. Fact or factitious? A psychobiological study of authentic and simulated dissociative identity states. PLoS One 2012; 7: e39279.

2 Şar V, Dorahy MJ, Krüger C. Revisiting the etiological aspects of dissociative identity disorder: a biopsychosocial perspective. Psychol Res Behav Manag 2017; 10: 137-46.

3 Dalenberg CJ, Brand BL, Loewenstein RJ, Frewen PA, Spiegel D. Inviting scientific discourse on traumatic dissociation: progress made and obstacles to further resolution. Psychol Inj Law 2020; 13: 135-54.

4 Nemeroff CB. Paradise lost: the neurobiological and clinical consequences of child abuse and neglect. Neuron 2016; 89: 892-909.

5 Reinders AATS, Marquand AF, Schlumpf YR, Chalavi S, Vissia EM, Nijenhuis ERS, et al. Aiding the diagnosis of dissociative identity disorder: pattern rec ognition study of brain biomarkers. Br J Psychiatry 2019; 215: 536-44.

6 Chalavi S, Vissia EM, Giesen ME, Nijenhuis ERS, Draijer N, Cole JH, et al Abnormal hippocampal morphology in dissociative identity disorder and posttraumatic stress disorder correlates with childhood trauma and dissociative symptoms. Hum Brain Mapp 2015; 36: 1692-704.

7 Reinders AATS, Chalavi S, Schlumpf YR, Vissia EM, Nijenhuis ERS, Jäncke L, Veltman DJ, Ecker C. Neurodevelopmental origins of abnormal cortical morphology in issociative identity disorder. Acta Psychiatr Scand 2018, 137: $157-170$ 